\title{
Improving the operation quality of technical systems using information theory models
}

\author{
Aleksander Dulesov ${ }^{1}$, Denis Karandeev ${ }^{1, *}$, and Natalia Dulesova ${ }^{2}$ \\ ${ }^{1}$ Katanov Khakass State University, 655017 Lenina ave. 92, Abakan, Russia \\ ${ }^{2}$ Khakass Technical Institute of Siberian Federal University, 655016 Komarova 15, Abakan, Russia
}

\begin{abstract}
Improving the operation quality of the technical system performance while designing and operating is considered through the application of information theory models. The models are based on the mathematical description of the probabilistic state of system elements and the possibility of determining the information entropy. To implement the model, the role of the stochastic behavior of a technical object is emphasized. This role determines its probability state. The basis for the determining of the entropy of object state based on a series of scientific and conceptual positions, developments of well-known scientists in connection with the problems of realization of physical processes. A mathematical model is proposed, that allows using the classical methods to determine the amount of information entropy and to use it to solve several problems: to choose the first preference structure with less uncertainty; to evaluate the behavior ("aging") of a system; to identify the problem areas of structures for the purpose of timely execution of equipment preventative maintenance; to construct the optimal system structure.
\end{abstract}

\section{Introduction}

In the course of complex systems evolution, essential attention from mathematicians and engineers is given on the problems of control and reliability of technical systems [1-3]. These problems are connected with the need of synergetics development and the theory improvement of self-organization systems $[4,5]$. The vastness of the existing theories and the complexity of systems don't fully allow to answer the question: how the unknown laws of nature influence the behavior of technical systems. The lack of information in the design of technical systems will affect the operation and the lack of accurate control will require additional resources. Identifying the same at the preliminary stages of the weak links in the system and their timely elimination in the course of operation must be carried out with the support of the solution of problem accounting information uncertainty using mathematical tools. They will allow calculating the quantitative and qualitative content of information about a system behavior. Such information can be used as a criterion for efficient management of system, acceptance management and design solutions. The implementation this a criterion is possible due to the developed mathematical tools allowing to remove uncertainty in the behavior of a technical object. Therefore, the solution of the mentioned

\footnotetext{
* Corresponding author: den dr house 1991@mail.ru
} 
problem associated with the creation of models, development of methods, algorithms and programs, which using will allow improving the operation quality of technical systems. Efficiency will be achieved, in addition to the use of known criteria (profitability, reliability, quality), but also by taking into account the uncertainty.

\section{The stochastic behavior of an object}

The stochastic behavior of an object - the real component of the full lifecycle of system operation. The random behavior of external and internal factors has always been a question of their detection to reduce the negative consequences [6]. One approach for solving these kind problems - a demand in the development of the modern technologies [7]. They will be based on totality of theories probability, statistics, physics, artificial intelligence, etc., that would allow "to build up" of work system so that the maximum to avoid negative consequences and to reduce risks to a minimum. The existing researches, scientific and practical experience in world practice (in other fields of knowledge, rather than technical) indirectly affect matters of lifecycle of technical systems $[8,9]$. Construction of models and development of methods must serve as an effective tool for performing the system analysis and synthesis capabilities of the technical system to perform given functions. To ensure proper functioning of these systems, it is necessary to determine the degree of removal of information uncertainty using analysis, information processing, forecasting and control.

The resolution the above-mentioned problem are connected with to solve the fundamental problem of creating tools for the processing of information in the conditions of uncertainty on a row of the directions. The first direction concerns the issues of determining the amount of information to the improving operation quality of technical systems. The answers to these questions can be obtained by systems analysis, information processing, forecasting parameters in the problem of constructing an optimal structure and efficient operation of the system of the network type. The task solution involves a system analysis with the detection of stochastic factors and regularities in the system behavior, to track the states of the system, processing the received information and subsequent forecasting. The problem of constructing the optimal structure is solved through the obtained amount of information and mathematical methods. The main object of research is the structure of system having presence of linkages between elements. Construction of the optimal structure will be based on a synthesis of criteria of cost-effectiveness, reliability and information uncertainty [10]. The criterion of cost-effectiveness includes efficiency of capital investments for creation or modernization of the system. The criterion ensuring the required level of reliability involves preliminary processing stochastic data and the accounting of two opposite qualitative states of an object (e.g. failures and working capacity). Paying attention to the demarcation information on qualitative character, creating the tools of demarcation of information on three or more states is required. This will allow to build methods for the determining of the amount of information for many states not only technical but also, for example, biological and social systems.

\section{Scientific novelty of the task and the existing state of research on this issue}

Scientific basis for most of the works of the quantitative information processing (from a line item of its uncertainty) most of all studies physical processes (statistical physics, quantum mechanics, thermodynamics), but not - social and biological processes. However, research in these fields can be used in the field of engineering knowledge, creation technologies analysis and synthesis of the unknown regularity of behavior of technical 
systems. This task is only a small part from all variety of the scientific researches directly related to the assessment and the impact of information uncertainty [11].

Taking into account the change of structure in the process of self-organization under the influence of a variety of changing factors, development and adoption of management decisions directed to supporting it in the future of its steady state through the removal of uncertainty. Such state of a system is typical e.g. for electricity consumption, when in the process of system operation to identify weaknesses in the system and to prevent negative consequences, leading to equipment failures. In the design of system structures, accounting of the information uncertainty through the probabilistic state elements will help to remove uncertainty, thus to make a choice in favor of the optimal structure.

Considering the existing researches in the field of analysis and synthesis of systems behavior from the perspective of the role of information in the process of their operation, it is necessary to select the essential contributions of several scientists and institutions: I. M Gurevich; A. Yu. Popkov; N. A. Kuznetsov; A. D. Ursul - the nature of the information [12]; B. B. Kadomtsev - information of physical processes; S. M. Korotaev and E. H. Liiv - philosophical concept of information and entropy [13]; D. S. Chernavskii - information in the biological and physical processes [14]; M. A. Basin - synergetics and entropic processes in living systems; V. Gagin - system synthesis; A. M. Panchenkov - paradigm entropy; A. P. Levich - entropy in thermodynamic systems; K. Tsallis - entropy in statistical physics [15]; W. R. Ashby - principle of the diversity of systems [16].

Existing researches about quantitative content of the uncertainty in a system, taking into account its dynamics, impel us to the need of the researches of regularities of information interaction between elements in complex technical systems. A preliminary review of the scientific literature presented by above-mentioned scientists and institutions has showed absence of the necessary of development: using measures of information uncertainty as a selection criterion effective structures and circuit design. Such development would allow to judge the state of a technical system to ensure the stable operation and efficient management. Solution of the problem is possible if we can to determine the measure of removal of uncertainty in case of the design and effective control based on the analysis and synthesis of information, creation of tools for its determination and involvement in the search of effective solutions.

\section{Approaches to improving the operation quality of technical systems}

Review of fundamental concepts, approaches, models and methods from statistical physics, information theory and entropy helped to develop a number of models and methods for determining the amount of entropy pertaining to the structure of the system $[17,18]$. The basis for creating tools are ideas of R. V. L. Hartley [19], A. N. Kolmogorov [20] and C. E. Shannon's classical theory [21] about obtaining the amount of information entropy. This entropy is expressed by a universal formula of the form $p \cdot \log p$, where $p-$ a probability of system element state. According to many scientists, this expression is promising, and the formula is the universal.

The structural reliability can be expressed through probabilistic feature of a network and thus it is possible to give it the characteristics of information uncertainty. The authors created a mathematical model for determining the information entropy of technical network structures, which allows to improve the operation quality of technical system by evaluating the structural reliability. The model partially implemented in [22-24], where the evaluation of structural reliability of a technical system is possible through the process of calculating the information entropy. Analytical calculation of reliability has been used in design and operation due to the strict requirements to provide high reliability of technical systems. 
Specifically, a method of full enumeration of states, a minimal paths and sections [25] and a fault tree analysis, etc. were widely adopted because of the use of block or logical diagrams. When solving such a problem and taking into account the division of elements states into operable and non-operable states, it is reasonable to differentiate entropy types according to this qualitative character [26]. Quantitative evaluation using analytical methods of entropy requires the construction of a model that identifies joint and conditional entropy when considering events in a system. That is why the technique offered in this work is based on modeling as well as on a method of making series and parallel structures equivalent with the aid of the structure decomposition as regard to a singular element.

To quantify entropy of $i$-th system element inclusive of its entropy division into qualitative components it is possible to use K. Shannon's equation [21]:

$$
H_{i}=-\left(p_{i} \log _{2} p_{i}+q_{i} \log _{2} q_{i}\right)
$$

The equation (1) is correct under condition that $p_{i}+q_{i}=1$, where $p_{i}$ and $q_{i}-$ the probabilities of operable and non-operable states of the $i$-th element. Value $H_{i}$ is measured by bit, and the logarithm base that equals 2 shows two opposite states.

Taking into account an independence condition of system elements operation, due to (1) one can get the formula for the total entropy in generalized type:

$$
H_{\Sigma}=-\sum_{i=1}^{N}\left[H\left(p_{i}\right)+H\left(q_{i}\right)\right]=-\sum_{i=1}^{N}\left(p_{i} \log _{2} p_{i}+q_{i} \log _{2} q_{i}\right)
$$

where $N$ - a number of elements, $H\left(p_{i}\right)$ and $H\left(q_{i}\right)$ - entropies of operable and non-operable states of $i$-th element. When all $p_{i}=q_{i}=0.5$, due to (2) one gets maximum entropy $H_{\max }=$ $H_{\Sigma}=N$. Equation (2) is necessary to monitor the correctness of the fulfilled calculations as the offered technique includes consideration of the joint states of the elements. In addition, equation (2) has a simplified view and not reflect probability of occurrence of joint events and elements states in the system structure. There is, however, a complete mathematical model to determine the amount of information entropy with its qualitative division for each of the selected states.

The model was tested in [22-26] and has the following features: - allows to determine the measure of the entropy with qualitative division into two opposite states of a system: operable and non-operable (for solving problems of system reliability); - takes into account a variety of elements states of the system structure; - allows to monitor the statics of the change of entropy; - don't exclude the possibility of further modernization, the development of new methods and the solving of task of the improving operation quality of technical systems. To implement the model, it is necessary to collect, pre-processing and the subsequent analysis of statistical or diagnostic data.

\section{Example of application of the model}

Let there be a simplest network structure of the technical system, presented in figure 1.

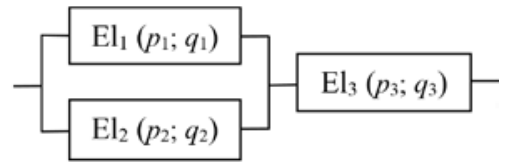

Fig. 1. A simplest structure of the technical system

This structure consists of 3 elements which are connected. Initial data are the probabilities of operable state of elements: $p_{1}=0.9 ; p_{2}=0.95 ; p_{3}=0.98$. 
The total entropy of the structure:

$$
H_{\Sigma}=-\sum_{i=1}^{3}\left(p_{i} \log _{2} p_{i}+q_{i} \log _{2} q_{i}\right)=0.897 \text { bit. }
$$

An entropy of a non-operable state of parallel-connected elements:

$$
H\left(q_{12}\right)=q_{1} H\left(q_{2}\right)+q_{2} H\left(q_{1}\right)=0.038 \text { bit. }
$$

An entropy of a non-operable state of equivalent structure:

$$
H(Q)=H\left(q_{12}\right)+H\left(q_{3}\right)+q_{12} H\left(p_{3}\right)+q_{3} H\left(p_{12}\right)=0.166 \text { bit. }
$$

An entropy of an operable state of parallel-connected elements:

$$
H\left(p_{12}\right)=H\left(p_{1}\right)+H\left(p_{2}\right)+p_{1} H\left(q_{2}\right)+p_{2} H\left(q_{1}\right)=0.717 \text { bit. }
$$

An entropy of an operable state of equivalent structure:

$$
H(P)=p_{12} H\left(q_{3}\right)+p_{3} H\left(q_{12}\right)=0.731 \text { bit } .
$$

The total entropy:

$$
H_{\Sigma}=H(Q)+H(P)=0.897 \text { bit } .
$$

The entropy an operable state has an advantage over the entropy of a non-operable state, thereby confirming the high level of structural reliability. In case of equality of all probabilities: $H_{\Sigma}=H(Q)+H(P)=1.825+1.175=3.0$ bit ,

where the entropy of the non-operable state prevails, which indicates an ineffective connection of elements in terms of structural reliability.

\section{Conclusion}

Proposed approaches to improve the operation quality of technical systems will allow not only to determine the amount of information, which is present at system, but also to consider various options of the structure to choose the most preferred among them (e.g., less uncertainty) and to evaluate the behavior ("aging") of the system in order timely to execute scheduled preventive maintenance of equipment. The obtained value of entropy and its components may be useful for generation tasks and adoption of effective solutions. In case of structure construction, the measure of information uncertainty can be used both in the form of restriction and target value of search of an optimal solution.

This research was supported by the grant "UMNIK" 2018 Program of the Russian Foundation for Assistance to Small Innovative Enterprises in Science and Technology (code № 0040353).

\section{References}

1. Charles E. Ebeling, An Introduction to Reliability and Maintainability Engineering, McGraw-Hill Companies, Inc., Boston, p. 486. (1997) 
2. I. A. Ushakov, Solving of optimal redundancy problem by means of a generalized generating function, Journal of Information Processing and Cybernetics archive, v. 24 (4-5), pp. 219-222. (1988)

3. O'Connor, Patrick D.T. Practical Reliability Engineering, John Wiley \& Sons, 4th ed. P. 431. (1998)

4. H. Haken, Synergetics: Introduction and Advanced Topics, Springer. (1983)

5. V. I. Shapovalov, N. V. Kazakov, The laws of synergetics and global trends, Social Sciences and modernity, v. 3, p. 141-148. (2002)

6. N. Wiener, Cybernetics: Or Control and Communication in the Animal and the Machine, Paris, (Hermann \& Cie) \& Camb. Mass. (MIT Press), 2nd revised ed. P. 232. (1961)

7. V. A. Alexandrov, A. V. Boyarshinov and P. Z. Wilinzon, Technics and technology in XXI century: modern state and prospects of development, Novosibirsk: CRNS Of Sibprint., P. 160. (2009)

8. Reda Farag, Haldar Achintya, A novel reliability evaluation method for large engineering systems, Ain Shams Engineering Journal, pp. 1-13. (2016)

9. G. Sankaraiah, Reddy Y. Raghunatha Design and optimization of an Integrated Reliability redundancy system with multiple constraints, 2nd International Conference on Reliability, Safety and Hazard - Risk-Based Technologies and Physics-of-Failure Methods (ICRESH), Mumbai, pp. 118-122. (2010)

10. A. S. Dulesov, D. Y. Karandeev Construction of optimal structure of technical system using "branch and bound", by criteria of profitability and reliability, Journal Safety \& Reliability of Power Industry., v. 33, pp. 56-59. (2016)

11. P. Shambadal, Development and Application of the Entropy Concept, Nauka, Moscow, P. 280. (1967)

12. A. D. Ursul, Nature of information: philosophical essay, Center of research global processes and sustainable development, v. 2, P. 231. (2010)

13. E. H. Liiv, Infodinamika. Infodynamics. Generalized entropy and negentropy, Tallinn: AO Juhisjelu, P. 200. (1998)

14. D. S. Chernavskii, Synergetics and information, M.: Science, P. 244. (2001)

15. C. Tsallis, Possible generalization of Boltzmann-Gibbs statistics, Journal of Statistical Physics, v. 52, pp. 479-487. (1988)

16. W. Ross Ashby, Principles of the Self-Organizing Dynamic System, In: Journal of General Psychology, v. 37, pp. 125-128. (1947)

17. Thomas M. Cover, A. Thomas Joy, Elements of Information Theory, New Jersey: Wiley and Sons, 2nd edition. P. 383. (2006)

18. L. Brillouin, Science and Information Theory, Academic Press, P. 347. (1956)

19. R. V. L. Hartley, Transmission of Information, Bell System Technical Journal, v. 7(3), pp. 535-563. (1928)

20. A. N. Kolmogorov, Three approaches to the quantitative definition of information, International Journal of Computer Mathematics., v. 1(1), pp. 3-11. (1965)

21. C. E. Shannon, Communication Theory of Secrecy Systems, Bell System Tech. J., v. 28, pp. 656-715. (1949)

22. A. S. Dulesov, D. Y. Karandeev and N. V. Dulesova, Reliability analysis of distribution network of mining enterprises electrical power supply based on measure of 
information uncertainty, IOP Conf. Series: Earth and Environmental Science, v. 87. pp. 1-6. (2017)

23. A. S. Dulesov, D. Y. Karandeev and T. G. Krasnova, The evaluation of the correlation between entropy and negentropy in the structure of a technical system, MATEC Web Conf. International Conference on Modern Trends in Manufacturing Technologies and Equipment (ICMTMTE), v. 129, pp. 1-4. (2017) DOI: https://doi.org/10.1051/matecconf/201712903005

24. A. S. Dulesov, D. Y. Karandeev and N. V. Dulesova, Optimal redundancy of radial distribution networks by criteria of reliability and information uncertainty, 3nd International Conference on Industrial Engineering, Applications and Manufacturing (ICIEAM), pp. 1-4. (2017) DOI: 10.1109/ICIEAM.2017.8076467

25. A. S. Dulesov, D. Y. Karandeev and N. N. Kondrat, Definition of amount of information entropy in structure of the technical system by method of the minimum ways, Modern high technologies., v. 2(3), pp. 425-429. (2016)

26. A. S. Dulesov, N. V. Dulesova and D. Y. Karandeev, Delimitation indicator of level of reliability of technical system on the qualitative character: entropy approach, Fundamental research, v. 2(3), pp. 477-481. (2016) 